been some successes in developing this kind of process, there are some distinct disadvantages: for example, using multiple components in the same reaction inevitably increases the possibility of unwanted side reactions. Controlling the chemoselectivity in multicomponent syntheses is therefore a challenging task, as is incorporating a suitable catalyst to achieve this enantioselectively. A team of researchers at Boston College, led by Amir Hoveyda, have now developed a simple multicomponent synthesis that achieves these goals.

In a one-pot process, the researchers carry out a chemoselective, site-selective and diastereoselective reaction using bis(pinacolato) diboron, a monosubstituted allene and a simple N-heterocyclic carbenecopper catalyst, to generate a boronsubstituted organocopper intermediate. This then participates in an equally selective substitution reaction with another component of the reaction, an allylic phosphonate, to produce - in high yields and with excellent selectivity - a highly versatile product bearing three separate functionalizable goups: a stereogenic carbon, a monosubstituted alkene and a $Z$-trisubstituted alkenylboron. Importantly, the copper catalyst used, identified through extensive screening efforts, is able to promote both enantioselective reactions, distinguishing between the double bonds of the allene and that of the allylic phosphonate, and ensuring the correct catalytic sequence takes place. This also helps to prevent the combination of reactants that would otherwise form various unwanted side-products.

To demonstrate the synthetic potential of these precursors for the synthesis of complex organic molecules, they were applied to the synthesis of the natural product rottnestol, a marine-sponge metabolite with promising antibiotic properties, and herboxidiene, an anticancer drug. Both target compounds were isolated with overall yields exceeding those of previous syntheses, and in gram-quantities. $J H$

\section{CROSS COUPLING}

\section{Substituting methoxy groups}

Angew. Chem. Int. Ed. http://dx.doi.org/10.1002/ anie.201402922 (2014)

Methoxy substituents on aromatic molecules are powerful directing groups, widely used in electrophilic aromatic substitution reactions and directed ortho-metallation reactions. And although their stability is useful for allowing them to survive harsh reaction conditions, it follows that removing them after they have served their purpose is challenging. Recent research on the use of nickel catalysts for methoxy removal has

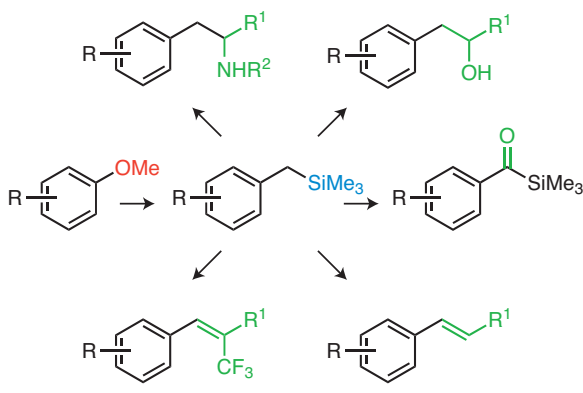

\section{blogroll $^{\text {i }}$}

\section{Hot and sweet}

Bloggers dip their sticky fingers into the foodie chemistry of honey and hot spices.

shown their utility in these reactions but there is still much room for improvement. Getting $s p^{3}$-hybridized carbon groups to replace methoxy is surprisingly difficult and currently limited to methyl groups. Similarly, introducing functional groups that are genuinely flexible with respect to further derivatization is also a challenge.

Magnus Rueping and co-workers from RWTH Aachen University encountered these issues during the course of a natural product synthesis but have now shown that methoxy groups can be easily replaced with functionalized aliphatic nucleophiles. Nickel catalysis was an obvious place to start, given the recent successes achieved in that area for the cleavage and replacement of methoxy groups. In particular, the team examined the effects of nickel catalysis with bifunctional $s p^{3}$-hybridized carbon nucleophiles. Grignard reagents performed poorly, but a silyl-group-bearing organolithium compound $\left(\mathrm{LiCH}_{2} \mathrm{SiMe}_{3}\right)$ was a good nucleophile and, after optimization, yields of $99 \%$ were achieved with a nickel cyclooctadiene catalyst. The reaction was rapid even at room temperature with $1 \mathrm{~mol} \%$ of catalyst. The scope for the aromatic coupling partner was broad with a number of functional groups (such as alcohols and amines) tolerated, and reactions could be scaled up without drops in yield.

The products - containing both silicon groups and benzylic hydrogen atoms were particularly amenable to further functionalization. Silyl groups are well known for increasing the acidity of neighbouring $\mathrm{C}-\mathrm{H}$ bonds; perhaps the best known use of this effect is in the Peterson olefination, which Rueping and co-workers were able to use to achieve alkene formation. In addition, fluoride-mediated reactions allowed the generation of nucleophilic $\mathrm{CH}_{2}$ and hence substitution of the trimethylsilyl group. Also, oxidation of the benzylic hydrogens gave acyl silanes, themselves useful building blocks. As such, the role of methoxy groups as precursors seems to have been greatly increased.

$E B$

Written by Enda Bergin, Claire Hansell, James Hennessy and Russell Johnson.
Summer is the time of year for barbecues and picnics, and the chemistry of food has clearly been on the minds of many in the blogosphere over the last few months. Leidamarie Tirado-Lee has written a fascinating blog post (http://go.nature. $\mathrm{com} / \mathrm{mxiN6q}$ ) for Helix Magazine at Northwestern University about the molecules that make food spicy. She admits to having almost developed an addiction for spicy food over recent years, despite being a late convert to the wonders of chilli peppers.

Tirado-Lee explains how capsaicinoids - the compounds in chilli peppers that cause the burning sensation - interact with the nerve cells on our tongue that normally respond to physical heat. These molecules effectively trick our brains into thinking we've been burned and, in response, our brains trigger the release of lots of lovely endorphins, which generate a natural 'high'. As she says, "next time you need a little pick-me-up consider giving in to the power of the chilli pepper and discover why chilliphiles have come to love the burn!"

Going from the hot to the sweet, Andy Brunning at the Compound Interest blog has been pondering (http://go.nature.com/8bFGeD) the chemistry of honey. He tells us that honey has such astonishing preservative properties that the oldest known sample was about 3,000 years old when it was discovered (in an Egyptian tomb) and it was still edible! After explaining how those clever bees produce honey, Brunning highlights the relevant sugar chemistry, pointing out the differences between sucrose, fructose and glucose. He then goes on to tell us how honey's low water content and carefully balanced acidity make it so good at inhibiting bacterial growth that it can even be used as a wound dressing.

Written by Kat Day, who blogs at http://thechronicleflask.wordpress.com 